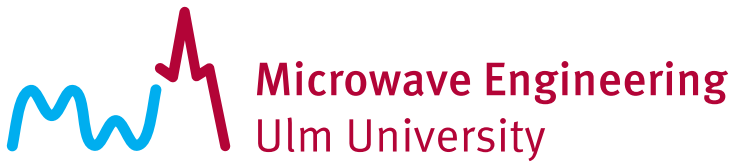

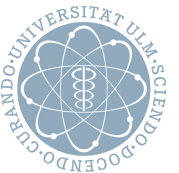

\section{A 160-GHz Radar Sniffer Probe for Honey Bee Detection}

Martin Geiger, Philipp Hügler, Thomas Galler, Torsten Bandel, Peter Ott, Wolf-Henning Rech, Thomas Walter, and Christian Waldschmidt 


\section{A 160-GHz radar sniffer probe for honey bee detection}

Geiger, Martin, Hügler, Philipp, Galler, Thomas, Bandel, Torsten, Ott, Peter, et al.

Martin Geiger, Philipp Hügler, Thomas Galler, Torsten Bandel, Peter Ott, WolfHenning Rech, Thomas Walter, Christian Waldschmidt, "A 160-GHz radar sniffer probe for honey bee detection," Proc. SPIE 11411, Passive and Active Millimeter-Wave Imaging XXIII, 1141103 (23 April 2020); doi:

$10.1117 / 12.2556145$

Event: SPIE Defense + Commercial Sensing, 2020, Online Only, California, United States 


\title{
A 160-GHz Radar Sniffer Probe for Honey Bee Detection
}

\author{
Martin Geiger ${ }^{\mathrm{a}}$, Philipp Hügler ${ }^{\mathrm{a}}$, Thomas Galler ${ }^{\mathrm{a}}$, Torsten Bandel ${ }^{\mathrm{b}}$, Peter Ott $^{\mathrm{c}}$, \\ Wolf-Henning Rech ${ }^{\mathrm{b}}$, Thomas Walter ${ }^{\mathrm{d}}$, and Christian Waldschmidt ${ }^{\mathrm{a}}$ \\ anstitute of Microwave Engineering, Ulm University, 89081 Ulm, Germany \\ ${ }^{\mathrm{b}}$ Pforzheim University of Applied Sciences, 75175 Pforzheim, Germany \\ ${ }^{c}$ Heilbronn University of Applied Sciences, 74081 Heilbronn, Germany \\ ${ }^{\mathrm{d}}$ Laboratory of Microtechnology, Ulm University of Applied Sciences, 89081 Ulm, Germany
}

\begin{abstract}
The use of radar systems is limited in some applications by spatial constraints or special thermal and environmental conditions. The spatial separation of the sensitive electronics and the more robust antenna by a flexible connection therefore opens up new applications. A 160-GHz radar system with a mechanically flexible front end fulfilling these requirements is proposed in this paper. The flexible front end is an extremely low loss dielectric waveguide feeding a dielectric elliptical lens antenna (28 dBi gain). The dielectric waveguide has dielectric losses of $4.5 \mathrm{~dB} / \mathrm{m}$ at $160 \mathrm{GHz}$ and is very flexible, allowing bending radii of down to $1.5 \mathrm{~cm}$ with negligible losses. The dielectric waveguide is fed by a $160-\mathrm{GHz}$ radar monolithic microwave integrated circuit (MMIC), which allows bandwidths of up to $20 \mathrm{GHz}$ for a high range resolution. The transition between MMIC and dielectric waveguide is realized with a rectangular-waveguide interface. The radar back end consists of a phased-locked loop (PLL) with standard components, an intermediate frequency (IF) signal conditioning part, and a Xilinx Zynq 7030 System-On-Module (SOM) with an FPGA and an ARM-based processor. The sampled signal is processed directly on the FPGA with a 2D Fourier transform and is available as a UDP stream with an update rate of up to $15 \mathrm{~Hz}$. In addition, a camera image is taken for each radar measurement. The presented system is used to detect and measure the flight behavior of honey bees. The electronics are housed in a building whereas the flexible dielectric waveguide allows the antenna to be placed anywhere around the beehive, where it is exposed to environmental conditions.
\end{abstract}

Keywords: chirp-secquence radar, dielectric waveguide, flexible radar sensor, lens antenna, millimeter wave, MMIC, MMIC-to-waveguide transition, radar sniffer probe

\section{INTRODUCTION}

Due to the progress in the silicon-germanium ( $\mathrm{SiGe}$ ) technology it is possible to produce radar sensors in large numbers at low cost. With compact and highly integrated monolithic microwave integrated circuits (MMICs) at frequencies above $100 \mathrm{GHz}$, a resolution in the millimeter range can be achieved. Therefore, radar sensors are very popular for distance and velocity measurements in various industrial applications such as level measurement, ${ }^{1}$ in the automotive industry, ${ }^{2,3}$ or for navigation in smoky rooms. ${ }^{4,5}$ Due to the robustness of the sensor concept against weather or lighting conditions as well as in dusty, smoky, and humid environments, the sensors can be used outdoors without restrictions. But the sensitive electronics require an appropriate housing, limiting the usage of radar sensors, particularly in close-range applications or in measurement environments with spatial constraints.

In order to avoid these limitations the robust and compact antenna front end can be separated from the sensitive electronics using flexible sensor heads like known from optics ${ }^{6}$ or from oscilloscope probes. Additionally, such a flexible sensor head provide the opportunity to measure at almost any desired position. The separation between the radar back end and the MMIC could be done by simple cables suitable for frequencies around a few MHz. Thus, the dimensions of the front end could be reduced, however, the MMIC still needs a robust housing.

Further author information:

M.G.: E-mail: martin-2.geiger@uni-ulm.de

Passive and Active Millimeter-Wave Imaging XXIII, edited by David A. Wikner,

Duncan A. Robertson, Proc. of SPIE Vol. 11411, 1141103 - (c) 2020 SPIE

CCC code: $0277-786 \mathrm{X} / 20 / \$ 21 \cdot$ doi: $10.1117 / 12.2556145$

Proc. of SPIE Vol. 11411 1141103-1

Downloaded From: https://www.spiedigitallibrary.org/conference-proceedings-of-spie on 28 Apr 2020 Terms of Use: https://www.spiedigitallibrary.org/terms-of-use 


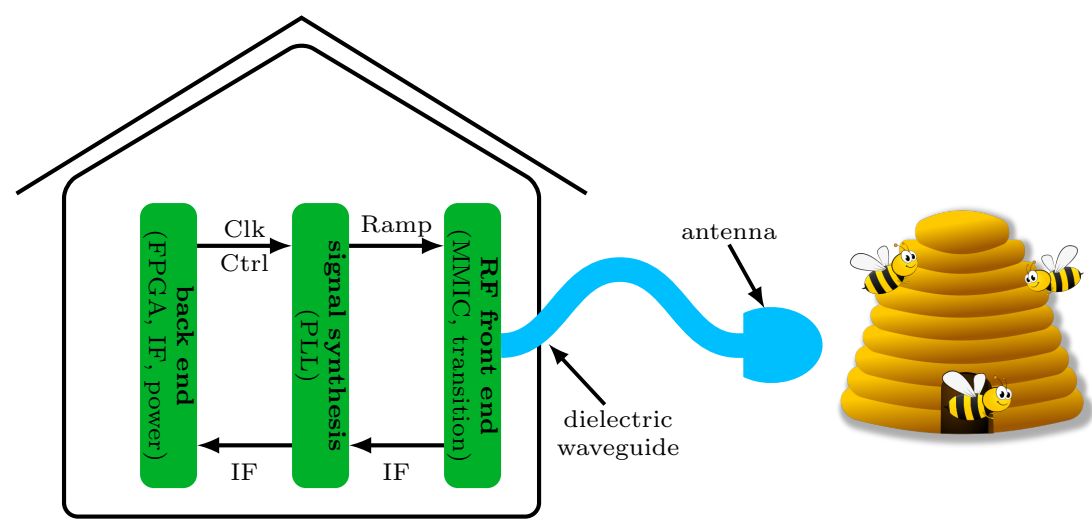

Figure 1. System concept of the $160-\mathrm{GHz}$ radar sensor with flexible front end.

A flexible antenna as a sensor head is more compact and in addition does not require a housing. However, a low-loss and flexible cable is required to feed the antenna at frequencies above $100 \mathrm{GHz}$. Microstrip lines are available on flexible substrate but they have high losses and need bond transitions from MMIC to the printed circuit board (PCB). Standard waveguides in this frequency range like metallic rectangular waveguides have less losses but are rigid. Another option is to apply dielectric waveguides used for frequencies above $100 \mathrm{GHz}$ in measurement setups,${ }^{7}$ communication systems,${ }^{8-10}$ and radar systems. ${ }^{11}$ Dependent on the used material, these waveguides are flexible, have low losses, and are robust against harsh environmental conditions such as humidity damaging the electronics.

In this paper a system concept for a $160-\mathrm{GHz}$ radar is presented, with the antenna separated from the MMIC and the back end electronics by a flexible dielectric waveguide. The system provides a live user data protocol (UDP) stream with the 2D Fourier transform (FT) radar data and thus enables live measurements for close-range scenarios with spatial constraints such as the observation of a beehive. At first, the system concept with front end and back end is explained. In Section 3 the individual components from the antenna to fieldprogrammable gate array (FPGA) are described in detail. The measurement setup and the results are presented in Section 4.

\section{SYSTEM CONCEPT}

The radar system presented consists of the radio frequency $(\mathrm{RF})$ front end with a mechanically flexible antenna and a corresponding back end, which processes the received signals and delivers the result of a 2D FT with an update rate of $15 \mathrm{~Hz}$. The components are built on individual PCBs for the back end (FPGA, intermediate frequency (IF) signal conditioning, power), signal synthesis, and the RF front end as a stack like shown in Figure 1.

The Xilinx Zynq Z-7030 FPGA is used to control the complete system. The FPGA not only programs the individual components such as IF amplifier, phased-locked loop (PLL) and MMIC, but also samples and processes the received signal. The PLL feeds a ramp signal to the front end consisting of a 160-GHz radar MMIC with transition to a metallic waveguide. This feeds a dielectric waveguide, which is very low-loss and mechanically flexible. Thus, the spatial separation of the antenna and the electronics is feasible. Since the target, the beehive, is located outdoors and is exposed to different weather conditions, the antenna must also be located outdoors. The remaining system electronics can be stored safely inside the building.

\section{COMPONENTS}

In the following section, the individual components are explained in more detail. At first, the front end with MMIC, transition, and dielectric waveguide are explained. Then, the back end with signal synthesis, IF signal conditioning, and the processing in the FPGA are described. 


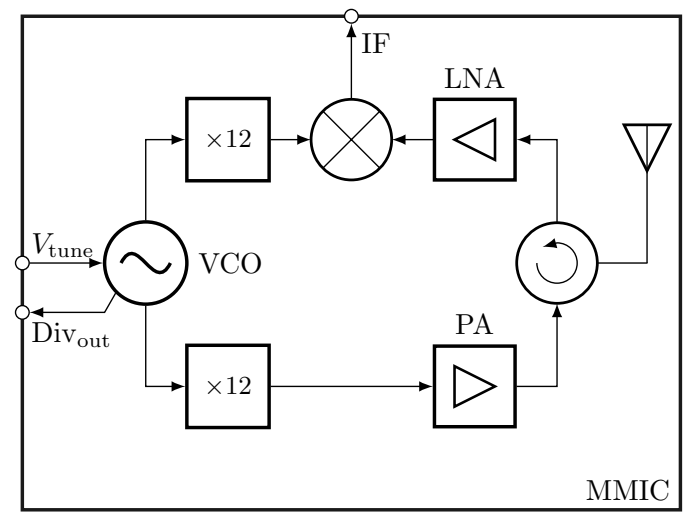

Figure 2. Block diagram of the MMIC.

\subsection{Front End}

The front end is based on a monostatic $160-\mathrm{GHz}$ radar MMIC. The signal couples via a resonant superstrate antenna on glass into a short dielectric waveguide. The tapered dielectric waveguide radiates the signal in a widened metallic waveguide which partially surrounds it. A mode converter feeds the flexible dielectric waveguide front end, which is terminated with an elliptical lens antenna. MMIC and flexible front end are decoupled to avoid mechanical stress.

\subsubsection{0-GHz radar MMIC}

The MMIC is a monostatic silicon-germanium radar chip with integrated voltage controlled oscillator (VCO) and a multiplier architecture as shown in Figure 2. The VCO can be tuned between $12.5 \mathrm{GHz}$ and $14.25 \mathrm{GHz}$ using the tuning voltage $V_{\text {tune }}$, resulting in a transmit frequency between $150 \mathrm{GHz}$ and $170 \mathrm{GHz}$ and a high range resolution. For the feedback ( $\operatorname{Div}_{\text {out }}$ ) to an external PLL module, the frequency is divided to ensure that only frequencies between $2.5 \mathrm{GHz}$ and $3.75 \mathrm{GHz}$ need to be routed on PCB.

For the monostatic front end a Tx-/Rx coupler is required, which is implemented as a compact rat-race coupler. ${ }^{12}$ With the integrated on-chip antenna, lossy bond transitions to PCB are avoided and the chip area extends to $1.0 \mathrm{~mm} \times 1.9 \mathrm{~mm}$.

\subsubsection{Transition from MMIC to metallic waveguide}

Since the system is intended to be permanently usable, a mechanically robust setup is required. For this purpose, a short metallic waveguide is used between MMIC and dielectric waveguide. It is mechanically decoupled from the MMIC avoiding that external stress affects the functionality. In addition, the dielectric waveguide, which is fed by a mode converter, is fixed mechanically.

For an efficient signal decoupling of the MMIC a transition as shown in Figure 3a is proposed. On the $\mathrm{SiO}_{2}$ back end of the MMIC a short-circuited $\lambda / 4$-patch is fed by a microstrip line. On top of the coupling structure a quartz glass with a metallized $\lambda / 2$-patch is positioned. ${ }^{13,14}$ The resonant patch excites the fundamental $\mathrm{HE}_{11}$ mode in a cylindrical dielectric waveguide made of acryl glass. The dimensions of the $\lambda / 4$-patch $(170 \mu \mathrm{m} \times 220 \mu \mathrm{m})$ and the quartz glass length $l_{r}$ determine the center frequency of the transition.

The transition to the metallic waveguide is realized with a non-resonant coupling by tapering the dielectric waveguide end to a rod antenna and transmitting the signal over a short free-space distance. A horn antenna with inverse taper is used as the receiving element. The two components can be approached to each other to prevent radiation and to minimize the free-space distance. The slope angle of the horn and the taper angle are identical in order to achieve a minimum attenuation.

The realized transition is shown in Figure $4 \mathrm{~b}$ and the S-parameters were determined. For the dimensions in Figure $3 \mathrm{~b}$ the minimum measured insertion loss is $3.1 \mathrm{~dB}$ at $147.1 \mathrm{GHz}$ as depicted in Figure $4 \mathrm{a}$. The reflection coefficient at the rectangular waveguide interface $\left(\mathrm{s}_{22}\right)$ is below $-10 \mathrm{~dB}$ from approximately $141 \mathrm{GHz}$ to $163 \mathrm{GHz}$. 


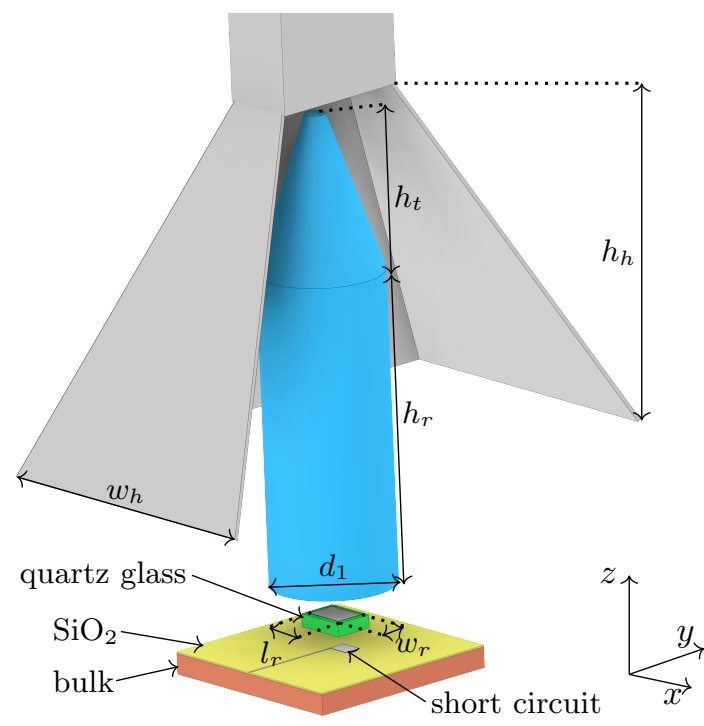

(a)

\begin{tabular}{c||c|c|c}
\multirow{2}{*}{ quartz glass } & $w_{r}$ & $l_{r}$ & $h_{q}$ \\
\cline { 2 - 4 } & 280 & 400 & 127 \\
\hline \hline \multirow{2}{*}{$\operatorname{rod}$} & $h_{r}$ & $h_{t}$ & $d_{1}$ \\
\cline { 2 - 4 } & 3700 & 1500 & 1200 \\
\hline \hline \multirow{2}{*}{ horn } & $w_{h}$ & $l_{h}$ & $h_{h}$ \\
\cline { 2 - 4 } & 3500 & 5690 & 4130
\end{tabular}

(b)

Figure 3. (a) Perspective view of a 3D model of the transition from MMIC to metallic waveguide and (b) the respective dimensions in $\mu \mathrm{m}$.

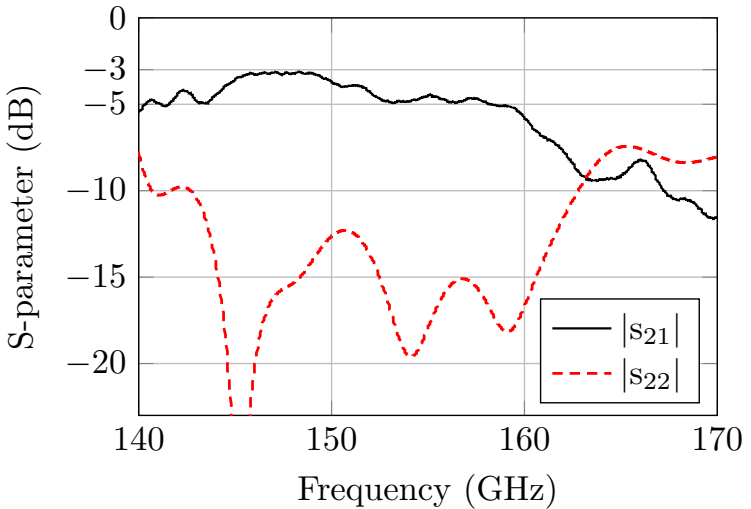

(a)

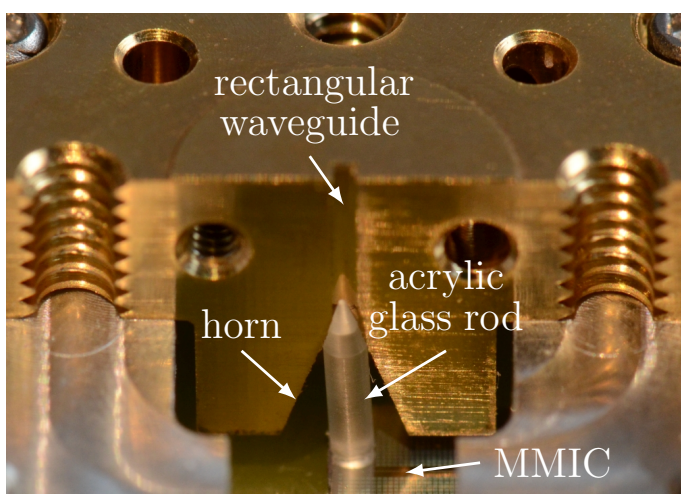

(b)

Figure 4. (a) Measured S-parameters of (b) the realized transition from MMIC to metallic waveguide. The rectangular waveguide is cut in the middle for the photo.

\subsubsection{Dielectric waveguide and antenna}

The dielectric waveguide is the flexible component of the front end and thus of great significance for the radar system. The electrical and mechanical properties like losses, flexibility, dispersion, and shielding of the dielectric waveguide are discussed in detail in this section.

The properties of the dielectric waveguide depend on the field distribution, which changes depending on the guided mode and the frequency as well as on the dimensions and the material permittivity of the waveguide. In the proposed system a single-mode waveguide with the fundamental $\mathrm{HE}_{11}$ mode is used, having field components both in the dielectric and in the air ${ }^{16}$ as shown in Figure 5a. By increasing the frequency, the dimensions, or the permittivity, the field density in the dielectric material increases and consequently, the dielectric losses and the attenuation rise. A further loss mechanism of the dielectric waveguide is the radiation at field disturbances or in bends. It behaves in contrary to the dielectric attenuation and is reduced for the above mentioned assumptions.

The designed rectangular dielectric waveguide with a cross-section of $648 \mu \mathrm{m} \times 1295 \mu \mathrm{m}$ is made of highdensity polyethylen $\left(\mathrm{HDPE}, \varepsilon_{r}=2.25, \tan \delta=3.1 \cdot 10^{-4}\right)$. The design is a trade-off between a minimum of losses 


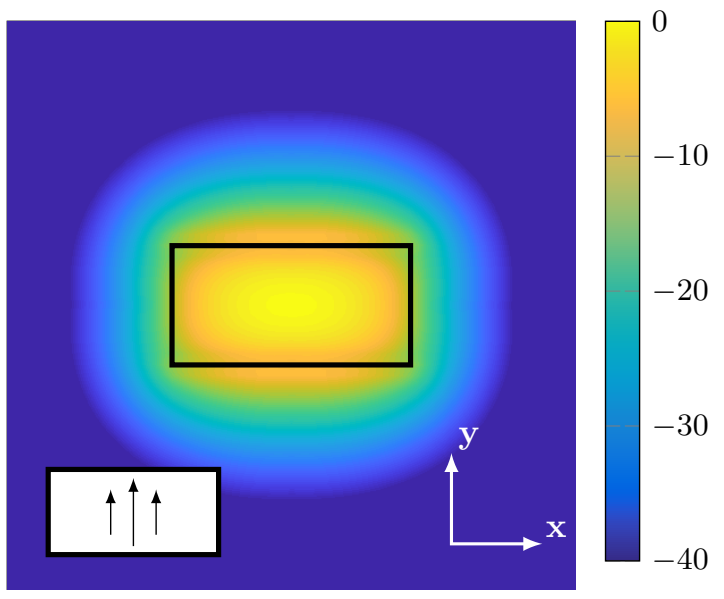

(a)

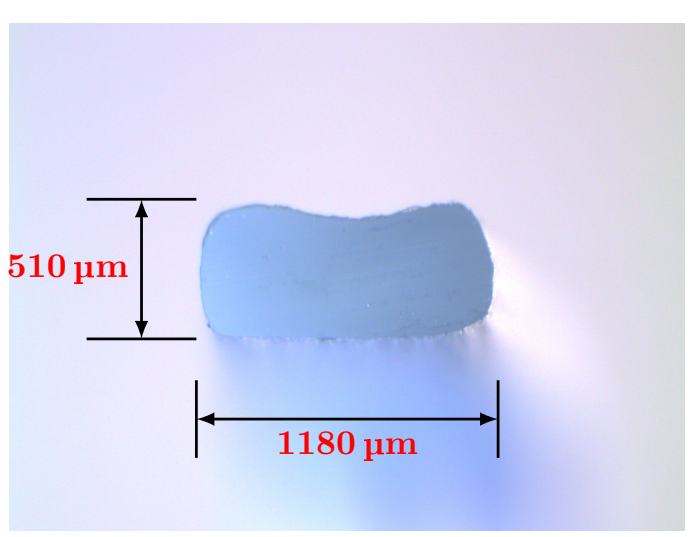

(b)

Figure 5. (a) E-field amplitude distribution of the $\mathrm{HE}_{11}$ mode in the dielectric waveguide and (b) the cross-section of the extruded dielectric waveguide. ${ }^{15}$

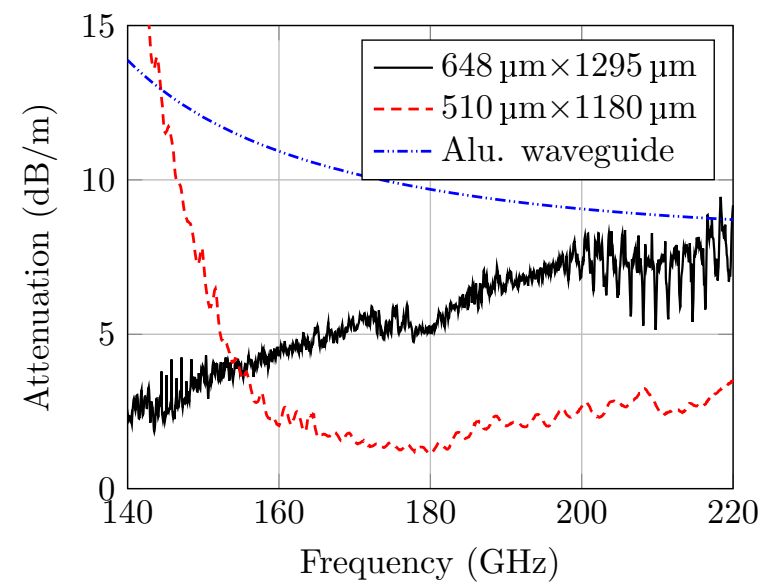

(a)

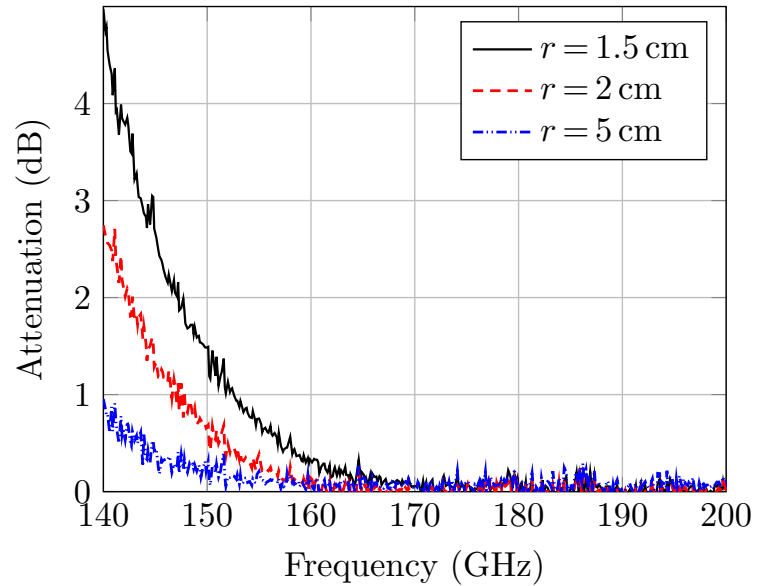

(b)

Figure 6. (a) Measured attenuation of the straight dielectric waveguides with different dimensions in comparison to the simulated losses of an aluminum waveguide. (b) Measured radiation losses in a $90^{\circ}$ bend for different radii $r$.

and a maximum of mechanical flexibility. The measured attenuation for a straight dielectric waveguide in a back-to-back measurement with different lengths for this waveguide dimensions is around $4.5 \mathrm{~dB} / \mathrm{m}$ at $160 \mathrm{GHz}$ and increases with higher frequencies as shown in Figure $6 \mathrm{a} .{ }^{11}$ In comparison to the simulated losses of an aluminum waveguide, ${ }^{17}$ the losses are significantly lower. Since a dielectric waveguide with a length above $1 \mathrm{~m}$ is required for the measurement setup, the used waveguide was fabricated by extrusion. Due to manufacturing tolerances the waveguide dimensions are slightly smaller $(510 \mu \mathrm{m} \times 1180 \mu \mathrm{m}$, cf. Figure $5 \mathrm{~b})$ and the attenuation is different. ${ }^{15}$ Especially in the lower frequency range, significant radiation losses occur at the mode transformers in the measurement setup. Above $155 \mathrm{GHz}$, however, the losses are lower due to the changed field distribution.

Since the waveguide must be protected outdoors, it requires a protective coating. The coating should not disturb the field distribution to avoid further losses, which demands for a material with a permittivity around $\varepsilon_{r}=1$. For this purpose either a low density foam like Rohacell or HDPE foam is used. ${ }^{18,19}$ For spacings between the dielectric waveguide and the outer edge of the foam exceeding several millimeters, an additional protective coating with a thin metal foil or a more robust plastic can be applied.

A further design aspect which has to be considered is the dispersion in the dielectric waveguide. ${ }^{15}$ Due to the dispersion the received signal is affected by the frequency-dependent group delay and the target peak in the 


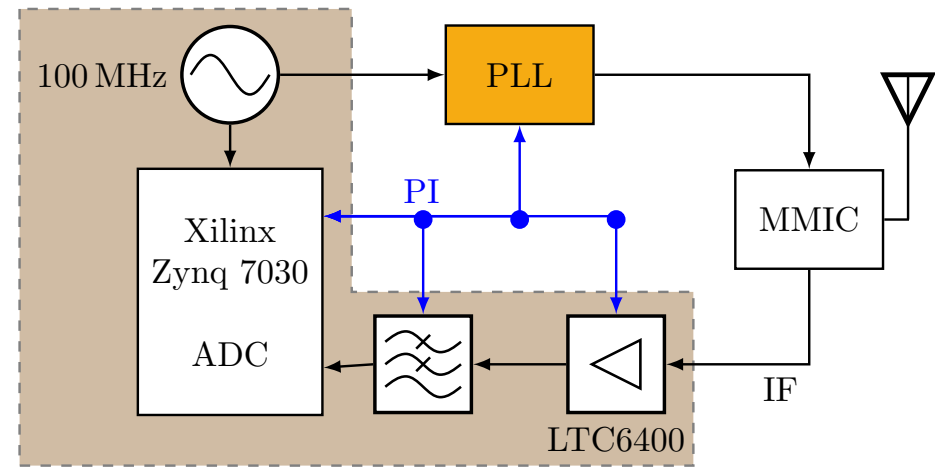

Figure 7. Block diagram of the radar system with its individual components.

radar response is broadened. This slightly deteriorates the range resolution to approximately $1.5 \mathrm{~cm}$ but does not restrict the bee detection. In addition, flying bees usually have a distance of more than $1.5 \mathrm{~cm}$ and can be separated by velocity.

For the detection of the bees, only a narrow field of view and a high gain is required. Therefore, an ellipsoidal lens antenna as proposed in [20] is used. The antenna is made of HDPE and is fed in its focal point by plugging it on the end of the dielectric waveguide. With a diameter of $1.5 \mathrm{~cm}$ a gain of $28 \mathrm{dBi}$ and a $3 \mathrm{~dB}$ angular width of $6.8^{\circ}$ is achieved.

\subsection{Back End}

To operate the MMIC as a frequency modulated continuous wave (FMCW) radar, a ramp signal (rampext) is fed to the MMIC, which is provided by an external PLL with VCO. The PLL module is controlled by the Xilinx Zynq 7030 FPGA as shown in Figure 7. The components (low-pass filter, amplifier) in the IF signal path are also programmed via the programmable interface (PI) using the Zynq 7030. All the components for the signal synthesis and IF signal processing are explained in the following sections.

\subsubsection{Signal synthesis and conditioning}

For the ramp generation with the PLL module several components are needed. The fractional-N PLL LMX2492 with the VCO HMC588 feeds the ramp signal with a frequency between $12.5 \mathrm{GHz}$ and $13.5 \mathrm{GHz}$ to the MMIC. The reference signal for the PLL is provided by a 100-MHz oscillator, also serving as a reference for the FPGA and its integrated components. An active loop filter is designed in standard feedback configuration ${ }^{21}$ with a cut-off frequency of $1 \mathrm{MHz}$.

In the IF signal path, the signal is amplified and filtered to lower the influence of quantization noise and to avoid aliasing. The operational amplifier LTC6400 with a fixed gain of $40 \mathrm{~dB}$ and a low-pass filter with a cut-off frequency of $55 \mathrm{MHz}$ are used.

\subsubsection{Signal sampling and processing}

The analog front end of the Zynq is a 14-bit analog-digital converter (ADC) with a sample rate of $100 \mathrm{MS} / \mathrm{s},{ }^{22}$ and the sampled signal is sent to the Zynq system via a JESD204b interface. An intellectual property (IP) core controls the data stream and sends it ramp by ramp as a block to the first signal processing unit. Both IP core and ADC are clocked by the external $100-\mathrm{MHz}$ reference clock to provide coherence to the analog front end with the PLL.

For the detection of a target in range and velocity direction a 2D FT with windowing in both dimensions needs to be implemented on the Zynq 7030. The windowing and the FT in range dimension is applied to the data stream of the incoming data from the control IP core as a Pipeline-Streaming-FT for each ramp. The FT results are temporarily stored in the RAM until all FT data of the individual ramps of the ramp block are available. Subsequently, the windowing and the FT are carried out to obtain the velocity information of the target. The processed data can be visualized by a web server or exported via an UDP interface. 


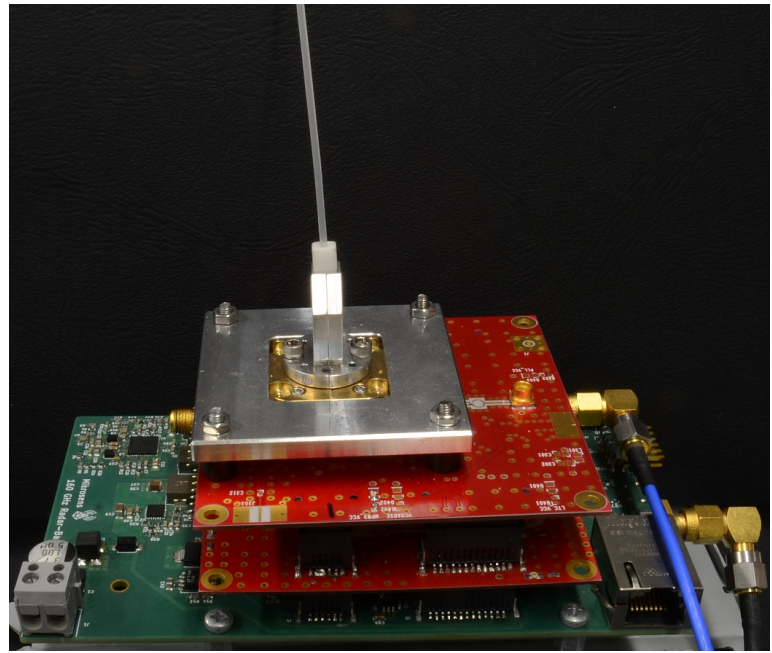

(a)

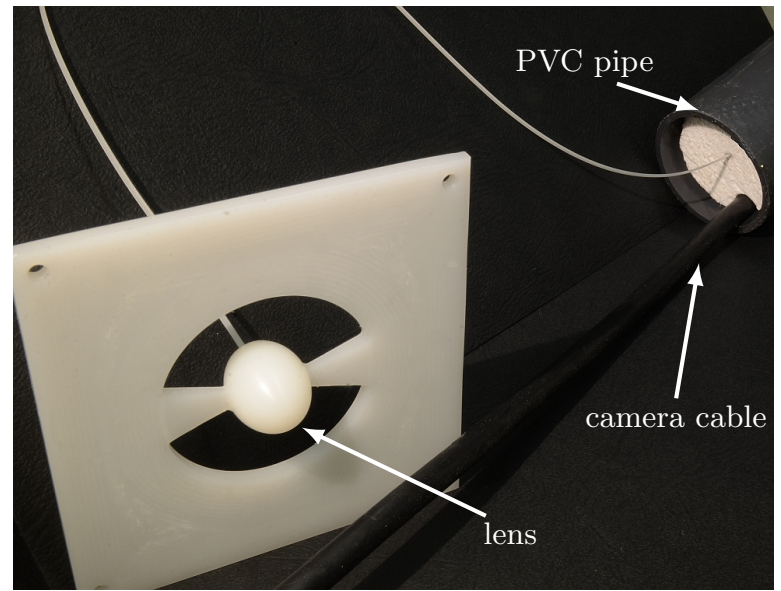

(b)

Figure 8. Realized radar system with (a) the radar stack and (b) the flexible front end with antenna and PVC pipe.

In addition to the provided radar data of the measured scenario, a camera image is stored for each ramp block to visualize the scenario and to compare measured radar data and the measurement scenario.

\section{MEASUREMENTS}

The velocity measurement of honey bees ${ }^{23,24}$ and the tracking of them using radar sensors ${ }^{25,26}$ is still a current research topic. The measurements are intended to demonstrate the detectability of bees with the presented system and to determine the flight velocities of bees around the hive. The measurement setup as well as measurement results are shown in the following sections.

\subsection{Measurement Setup}

The entire radar system is built up as a stack consisting of several PCBs with the respective functionalities and is located in a protected housing within the building. The radar MMIC with the transition to the dielectric waveguide is placed on the uppermost PCB as shown in Figure 8a. The dielectric waveguide has a length of $1.15 \mathrm{~m}$ and is guided through an opening to the beehive. To protect the dielectric waveguide, a polyvinyl chloride (PVC) pipe with Rohacell brackets is used (cf. Figure 8b). Additionally, a cable is routed to control the camera and receive the camera data. The dielectric waveguide is terminated by the lens antenna, which can be placed arbitrarily around the beehive. For measurements the beehive was observed with the antenna orientation both frontally to the entrance hole and laterally to the entry lane. The radar parameters used for the measurements are shown in Table 1.

Table 1. Radar parameters for the measurements.

\begin{tabular}{c|c||c|c} 
sampling frequency $f_{s}$ & $100 \mathrm{MHz}$ & number of ramps $L$ & 256 \\
\hline RF bandwidth $B$ & $9 \mathrm{GHz}$ & ramp duration $T_{\mathrm{ramp}}$ & $85 \mu \mathrm{s}$ \\
\hline & & ramp repetition interval $T_{\mathrm{RRI}}$ & $170 \mu \mathrm{s}$ \\
\hline range resolution $\Delta R$ & $1.67 \mathrm{~cm}$ & velocity resolution $\Delta v$ & $0.022 \mathrm{~m} / \mathrm{s}$ \\
\hline maximum range $R_{\max }$ & $63.71 \mathrm{~m}$ & maximum velocity $\left|v_{\max }\right|$ & $2.76 \mathrm{~m} / \mathrm{s}$
\end{tabular}




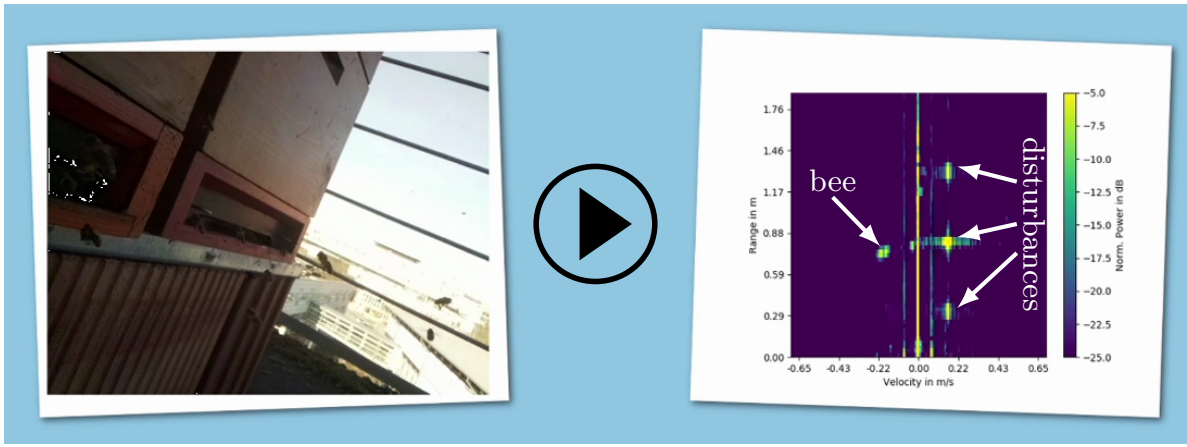

Figure 9. Video 1, range-Doppler diagram of the radar measurement with the antenna orientation laterally to the entry lane and camera view on the measurement scenario. http://dx.doi.org/10.1117/12.2556145

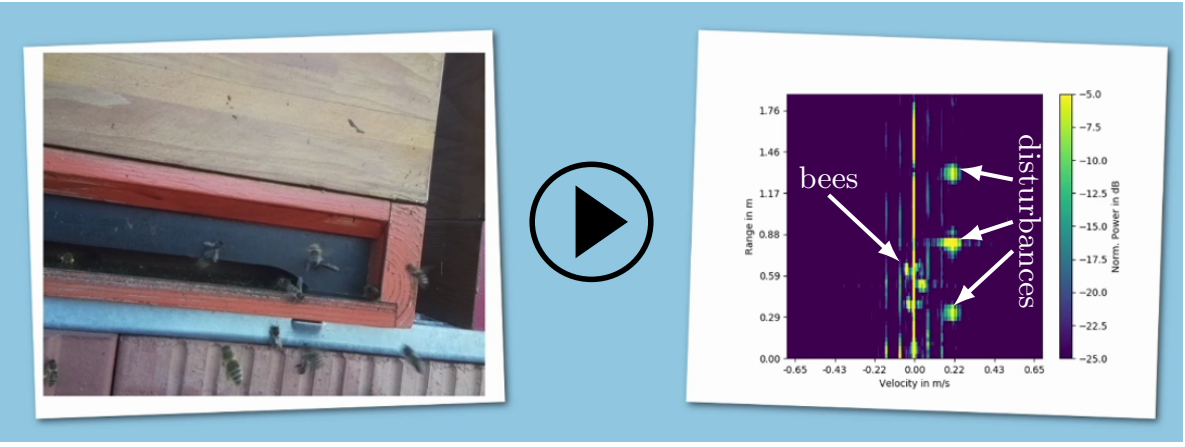

Figure 10. Video 2, range-Doppler diagram of the radar measurement with the antenna orientation frontally to the entrance hole and camera view on the measurement scenario. http://dx.doi.org/10.1117/12.2556145

\subsection{Measurement Results}

During the first measurement the beehive was observed laterally by the radar sensor, as shown in Figure 9. The entry lane of the bees was not exactly parallel to the propagation direction of the wave, because the bees fly off the building behind the antenna. In the range-Doppler diagram the reflection of a flying bee is visible at a distance of $0.7 \mathrm{~m}$ and a velocity of $-0.2 \mathrm{~m} / \mathrm{s}$. In addition, constant disturbances caused by the sensor itself are present at $0.3 \mathrm{~m}, 0.75 \mathrm{~m}$, and $1.35 \mathrm{~m}$. They could be corrected by a calibration. During the time period of observation, bees were detected in the range between $0.25 \mathrm{~m}$ and $1.3 \mathrm{~m}$ with a absolute maximum velocity $\left|v_{\max }\right|$ of $0.3 \mathrm{~m} / \mathrm{s}$.

For the second measurement the antenna was oriented frontally to the beehive with a distance of $60 \mathrm{~cm}$ as shown in Figure 10. In the range-Doppler diagram the entrance hole is seen at a distance of approximately $60 \mathrm{~cm}$, since targets with velocity components caused by the bees in the hive are continuously measured. The arriving and departing bees are detected at lower distances with velocities between $-0.3 \mathrm{~m} / \mathrm{s}$ and $0.3 \mathrm{~m} / \mathrm{s}$. Furthermore, the similar disturbances as in Figure 9 are visible.

The same radial velocities are measured for both scenarios despite the orientation of the antenna to the entry lane is changed. This results from the flight behavior of bees in front of the hive. Instead of a straight flight path, the bees slow down and suddenly change the flight direction arbitrarily. Therefore, the maximum speed of up to $8 \mathrm{~m} / \mathrm{s}$ achievable by bees is not measured.

\section{CONCLUSION}

This paper presents a 160-GHz radar system with a flexible front end for the spatial separation of sensitive electronics and robust antenna front end in harsh environments. With the back end based on a Xilinx Zynq 7030 FPGA, the complete radar systems allows live measurements of range and velocity with an update rate of up to $15 \mathrm{~Hz}$, as demonstrated by the detection of bees. 
The radar front end is based on an MMIC with a bandwidth of $20 \mathrm{GHz}$ for a high range resolution. Via a transition from MMIC to the rectangular waveguide interface, a low-loss and flexible dielectric waveguide is fed. It is made of HDPE and is bendable with negligible losses down to a radius of $1.5 \mathrm{~cm}$. A high gain lens antenna $(28 \mathrm{dBi})$ is plugged on the end of the dielectric waveguide to focus the radiated signal. The received IF signal is sampled by the FPGA and the data processed with a 2D FT is provided on a web server or as an UDP stream.

The system was demonstrated for the detection of bees in front of their hive. They were clearly visible in the range-Doppler diagram up to a distance of $1.3 \mathrm{~m}$ at maximum velocities between $-0.3 \mathrm{~m} / \mathrm{s}$ and $0.3 \mathrm{~m} / \mathrm{s}$.

\section{ACKNOWLEDGMENTS}

This work was supported by the Ministry for Science, Research, and Arts Baden-Württemberg within the project ZAFH MikroSens.

\section{REFERENCES}

[1] Brumbi, D., "Low Power FMCW Radar System for Level Gaging," in [IEEE MTT-S International Microwave Symposium Digest], 3, 1559-1562 (June 2000).

[2] Hasch, J., Topak, E., Schnabel, R., Zwick, T., Weigel, R., and Waldschmidt, C., "Millimeter-Wave Technology for Automotive Radar Sensors in the $77 \mathrm{GHz}$ Frequency Band," IEEE Transactions on Microwave Theory and Techniques 60, 845-860 (Mar. 2012).

[3] Bauer, F., Wang, X., Menzel, W., and Stelzer, A., "A 79-GHz Radar Sensor in LTCC Technology Using Grid Array Antennas," IEEE Transactions on Microwave Theory and Techniques 61, 2514-2521 (June 2013).

[4] Kueppers, S., Cetinkaya, H., and Pohl, N., "A Compact $120 \mathrm{GHz}$ SiGe:C Based $2 \times 8$ FMCW MIMO Radar Sensor for Robot Navigation in Low Visibility Environments," in [European Radar Conference (EURAD)], 122-125 (Oct. 2017).

[5] Klenner, M., Zech, C., Hülsmann, A., Kühn, J., Schlechtweg, M., Hahmann, K., Kleiner, B., Ulrich, M., and Ambacher, O., "A Portable W-Band Radar System for Enhancement of Infrared Vision in Fire Fighting Operations," in [Millimetre Wave and Terahertz Sensors and Technology IX], Salmon, N. A. and Ahmed, S. S., eds., 9993, 45 - 53, International Society for Optics and Photonics, SPIE (2016).

[6] Kudo, H., Miyajima, K., Takahashi, D., Arakawa, T., Saito, H., Mitsubayashi, K., and Sawai, M., "Fiber Optic Bio-Sniffer (Biochemical Gas Sensor) Using UV-LED Light for Monitoring Ethanol Vapor with High Sensitivity \& Selectivity," in [IEEE Sensors], 1955-1958 (Oct. 2009).

[7] Weinzierl, J., Fluhrer, C., and Brand, H., "Dielectric Waveguides at Submillimeter Wavelengths," in [IEEE Sixth International Conference on Terahertz Electronics Proceedings], 166-169 (Sept. 1998).

[8] Dey, U. and Hesselbarth, J., "Building Blocks for a Millimeter-Wave Multiport Multicast Chip-to-Chip Interconnect Based on Dielectric Waveguides," IEEE Transactions on Microwave Theory and Techniques 66, 5508-5520 (Dec. 2018).

[9] Fukuda, S., Hino, Y., Ohashi, S., Takeda, T., Yamagishi, H., Shinke, S., Komori, K., Uno, M., Akiyama, Y., Kawasaki, K., and Hajimiri, A., "A 12.5+12.5 Gb/s Full-Duplex Plastic Waveguide Interconnect," IEEE Journal of Solid-State Circuits 46, 3113-3125 (Dec. 2011).

[10] De Wit, M., Ooms, S., Philippe, B., Zhang, Y., and Reynaert, P., "Polymer Microwave Fibers: A New Approach That Blends Wireline, Optical, and Wireless Communication," IEEE Microwave Magazine 21, 51-66 (Jan. 2020).

[11] Geiger, M., Hitzler, M., Saulig, S., Iberle, J., Hügler, P., and Waldschmidt, C., "A 160-GHz Radar With Flexible Antenna Used as a Sniffer Probe," IEEE Sensors Journal 17, 5104-5111 (Aug. 2017).

[12] Hitzler, M., Grüner, P., Boehm, L., Mayer, W., and Waldschmidt, C., "On Monostatic and Bistatic System Concepts for mm-Wave Radar MMICs," IEEE Transactions on Microwave Theory and Techniques 66, 4204-4215 (Sept. 2018).

[13] Hasch, J., Wostradowski, U., Gaier, S., and Hansen, T., "77 GHz Radar Transceiver with Dual Integrated Antenna Elements," in [German Microwave Conference Digest of Papers], 280-283 (Mar. 2010). 
[14] Hitzler, M., Saulig, S., Boehm, L., Mayer, W., and Waldschmidt, C., "MMIC-to-Waveguide Transition at $160 \mathrm{GHz}$ with Galvanic Isolation," in [IEEE MTT-S International Microwave Symposium (IMS)], 1-4 (May 2016).

[15] Geiger, M., Wegner, C., Mayer, W., and Waldschmidt, C., "A Wideband Dielectric Waveguide-Based 160GHz Radar Target Generator," Sensors 19(12) (2019).

[16] Marcatili, E. A. J., "Dielectric Rectangular Waveguide and Directional Coupler for Integrated Optics," The Bell System Technical Journal 48, 2071-2102 (Sept. 1969).

[17] Collin, R., [Foundations for Microwave Engineering], IEEE Press Series on Electromagnetic Wave Theory, Wiley (2001).

[18] Geiger, M. and Waldschmidt, C., "160-GHz Radar Proximity Sensor With Distributed and Flexible Antennas for Collaborative Robots," IEEE Access 7, 14977-14984 (2019).

[19] Distler, F., Oppelt, D., Schür, J., and Vossiek, M., "Design and Characterization of a Compact and Robust Shielded Dielectric Waveguide for mmW Applications," in [11th German Microwave Conference (GeMiC)], 375-378 (Mar. 2018).

[20] Geiger, M., Hitzler, M., Iberle, J., and Waldschmidt, C., "A Dielectric Lens Antenna Fed by a Flexible Dielectric Waveguide At $160 \mathrm{GHz}$," in [11th European Conference on Antennas and Propagation (EuCAP)], 3380-3383 (Mar. 2017).

[21] Banerjee, D., [PLL Performance, Simulation and Design], Dog Ear Publishing (2006).

[22] Kesel, F., Bandel, T., and Rech, W.-H., "RADAR Signal Processing on an Embedded System using the Xilinx Zynq Platform," in [Embedded World Conference], (Feb. 2019).

[23] Wenner, A. M., "The Flight Speed of Honeybees: a Quantitative Approach," Journal of Apicultural Research 2(1), 25-32 (1963).

[24] Baird, E., Srinivasan, M. V., Zhang, S., and Cowling, A., "Visual control of flight speed in honeybees," Journal of Experimental Biology 208(20), 3895-3905 (2005).

[25] Riley, J. R., Smith, A. D., Reynolds, D. R., Edwards, A. S., Osborne, J. L., Williams, I. H., Carreck, N. L., and Poppy, G. M., "Tracking bees with harmonic radar," Nature 379, 29-30 (1996).

[26] Tsai, Z., Jau, P., Kuo, N., Kao, J., Lin, K., Chang, F., Yang, E., and Wang, H., "A High-Range-Accuracy and High-Sensitivity Harmonic Radar Using Pulse Pseudorandom Code for Bee Searching," IEEE Transactions on Microwave Theory and Techniques 61, 666-675 (Jan. 2013). 